\title{
On some special properties of 'why' in syntax and prosody
}

\section{Bocci, Giuliano}

De Gruyter Mouton

2021-09

Bocci , G , Cruschina , S \& Rizzi , L 2021, On some special properties of 'why' in syntax and pÿprosody . in G Soare (ed.), Why is why Unique? Its syntactic and semantic properties . De Gruyter Mouton , Berlin , pp. 293-316 . https://doi.org/10.1515/9783110675160-011

http://hdl.handle.net/10138/334309

https://doi.org/10.1515/9783110675160-011

acceptedVersion

Downloaded from Helda, University of Helsinki institutional repository.

This is an electronic reprint of the original article.

This reprint may differ from the original in pagination and typographic detail.

Please cite the original version. 


\title{
On some special properties of why in syntax and prosody
}

\author{
Giuliano Bocci (University of Siena) \\ Silvio Cruschina (University of Helsinki) \\ Luigi Rizzi (Collège de France)
}

\section{Introduction}

Syntactic research over the last twenty years has uncovered numerous peculiarities in the syntactic behaviour of why, in comparison with other wh-elements (see Soare (this volume); cf. also the discussion in Stepanov \& Tsai 2008). An important difference, for instance, concerns word order. In some languages requiring subject inversion in wh-interrogatives, why is exceptional in that it allows the non-inverted order wh-subject-inflected verb (Rizzi 1997, 2001a). This can be illustrated by the contrast between the Italian equivalents of what and how on the one hand $(1 \mathrm{a}, \mathrm{b})$, and why on the other $(1 \mathrm{c})$ :

$\begin{array}{ccclll}\text { a. } & \text { * Che cosa } & \text { Gianni } & \text { dice } & \text { a } & \text { Piero } \\ \text { what } & \text { Gianni } & \text { says } & \text { to } & \text { Piero } \\ \text { b. } & \text { Come } & \text { Gianni } & \text { contatterà } & \text { Piero } \\ & \text { how } & \text { Gianni } & \text { contact.FUT.3SG } & \text { Piero } \\ \text { c. } & \text { Perché } & \text { Gianni } & \text { contatterà } & \text { Piero } \\ & \text { why } & \text { Gianni } & \text { contact.FUT.3SG } & \text { Piero } \\ & \text { 'Why will Gianni contact Piero?' } & \end{array}$

To account for this syntactic asymmetry Rizzi (2001a) proposed that, while other wh-elements are extracted from the IP, perché (why) is externally merged in the left periphery. More precisely, why is first merged in the Spec of Int(errogative), a dedicated left-peripheral position also hosting $s e$ (if), the marker of embedded yes/no questions. ${ }^{1}$ Whereas other wh-elements target the lower Foc(us) position, which in turn attracts the inflected verb endowed with $+Q$, $\mathrm{Int}^{\circ}$ is inherently endowed with $+\mathrm{Q}$, hence it does not trigger movement of a verbal element to the left periphery. This captures the absence of obligatory inversion with why.

\footnotetext{
${ }^{1}$ See Shlonsky and Soare (2011) for a revised version of this analysis, according to which why is externally merged in the left periphery, but in a lower dedicated functional layer. In this analysis, why reaches its final position in Spec of Int via movement. Crucially, this approach maintains that why is not extracted from the IP since it is first merged in the left periphery.
} 
The main aim of this paper is to show that, in Italian, why-questions are different from other wh-questions not only syntactically, but also prosodically. We argue that the same formal apparatus explaining the special syntactic behaviour of why can be used to also capture the prosodic peculiarities of why with respect to prominence distribution. In particular, we will discuss two phenomena: (i) subject inversion, and (ii) the assignment of main prominence, i.e. sentential stress and the nuclear pitch accent (henceforth, NPA). Our argument for the special status of why will be supported by experimental findings related to both phenomena. For subject inversion in why-questions, we will rely on the studies by Bocci \& Pozzan (2014) and Bianchi, Bocci \& Cruschina (2017), where the position of the subject in why-questions is tested also against information-structure conditions. As for prominence distribution in wh-questions, our starting point will be the experimental findings discussed in Bocci, Bianchi \& Cruschina (2020) on wh-questions introduced by bare wh-elements other than why. We will then present the results of our own experiment specifically aimed at testing prominence distribution in whyquestions as opposed to other types of wh-questions.

Prosodically, why-questions are different from questions with other wh-elements. Bocci, Bianchi \& Cruschina (2020) show that in wh-questions, with wh-elements other than why, the NPA is always assigned, in Italian, to the lexical verb and never to the wh-element itself (see also Marotta 2001). This somehow unexpected property is taken to be a reflex of the derivational history of the wh-element, in that in Italian the main prominence (i.e. the NPA and sentential stress) tracks the intermediate positions of the wh-element, which moves stepwise from a vP internal position to the $\mathrm{vP}$ edge, and then to the CP system. The $w h$-phrase is endowed with a [wh, focus] feature bundle, which the wh-phrase shares with every phase head that intervenes along its movement path. Prominence assignment to the verb is then a "memory" of this transition event, couched in formal featural terms.

A different pattern emerges with why: in direct interrogative sentences introduced by why, the main prominence typically falls on why itself, and not on the verb, as in the pattern observed with other wh-elements. We take this prosodic asymmetry to be directly linked to the different syntactic derivation of why, which, unlike other wh-elements, does not undergo cyclic movement from a clause internal position, but is externally merged in a left-peripheral dedicated position. $^{2}$

\footnotetext{
${ }^{2}$ In this paper, we will not discuss cases in which why is construed long distance with an embedded clause, an interpretation that is possible, for instance, in the following example, which can be interpreted as a question about the reason of the firing:

(i) Why did you say he was fired?

Cases like this inevitably involve movement. Since why in (i) moves through the CP phase of the embedded clause and the vP phase of the matrix clause, our analysis - to be presented in Section 6 - predicts that the prosodic
} 


\section{The syntax of Italian wh-questions}

Before turning to why-questions, let us first consider the general syntactic behaviour of whquestions. In Italian direct wh-questions with bare wh-elements other than why, neither subjects nor other constituents can intervene between the wh-phrase and the inflected verb - the subject, for instance, must occur postverbally (Calabrese 1982, Rizzi 1996, 2001a), as shown in (2): ${ }^{3}$

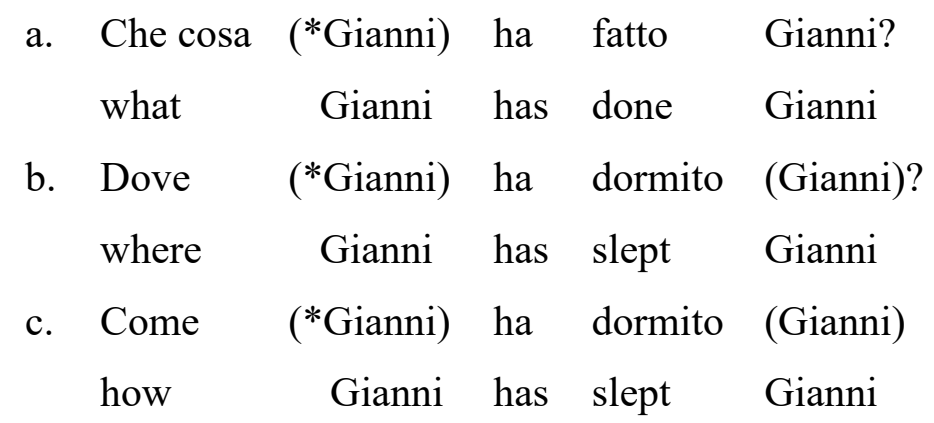

Unlike English, in Italian the subject does not necessarily undergo inversion: it can be dislocated to the left or simply omitted. In line with the previous literature, however, we use the term subject inversion to refer to this syntactic restriction. ${ }^{4}$

A second syntactic property discussed in Rizzi $(1997,2001 \mathrm{a})$ is that fronted foci are incompatible with a wh-phrase within the left periphery of the clause, irrespective of the linear order, as shown in (3). See the experimental evidence presented in Bocci, Rizzi, and Saito (2018). More generally, we can say that wh-phrases are incompatible with any narrow focus, insofar as this restriction also operates when the focus is in situ, as in the example in (4) (from Bocci 2013:19), at least in genuine direct wh-questions with an interrogative interpretation.

$$
\begin{array}{ccl}
\text { a. * A chi } & \text { QUESTO hanno detto? } \\
\text { to whom this } & \text { have.3PL said } \\
\text { c. * QUESTO a chi } & \text { hanno detto? } \\
\text { why } & \text { to whom } & \text { have.3PL said }
\end{array}
$$

\footnotetext{
system of Italian should keep trace of these intermediate steps in the derivation. We leave the discussion of these cases for future work.

${ }^{3}$ On the distribution of the subject in indirect questions, see Bocci \& Pozzan (2014) and Bocci \& Cruschina (2018).

${ }^{4}$ Some apparent exceptions are discussed in Cardinaletti (2007) where it is shown that specific types of adverbs, can indeed intervene between the wh-phrase and the verb. For simplicity, here we describe subject inversion in its traditional terms as an adjacency requirement and refer to her work for the relevant exceptions. Note also that in Italian, unlike Germanic languages, subjects cannot occur between the auxiliary and the verb in analytic forms (see, e.g., Rizzi 1996).
} 


$\begin{array}{clll}\text { * Quando hanno } & \text { consegnato } & \text { IL LIBRO a Leo? } \\ \text { to whom have.3PL given } & \text { the book to Leo }\end{array}$

Why-questions behave differently in this respect: they admit the non-inverted order whsubject-inflected verb, that is, they allow either a preverbal $(5 a)$ or a postverbal subject $(5 b)$. The two positions for the subject are not in free variation, but are sensitive to information structure conditions, much as in declarative sentences (cf. $\S 3$ below). Why-questions show a second relevant difference with respect to other wh-questions: they are compatible with a narrow focus, as shown in (6) (see Rizzi 1997, 2001a):

$\begin{array}{clll}\text { a. } & \text { Perché } & \text { Stefano } & \text { balla? } \\ \text { why } & \text { Stefano } & \text { dances } \\ \text { b. Perché } & \text { balla } & \text { Stefano? } \\ & \text { why } & \text { dances } & \text { Stefano }\end{array}$

'Why is Stefano dancing?'

(6)

$\begin{array}{lllll}\text { Perché } & \text { QUESTO } & \text { avremmo dovuto dirgli? } \\ \text { why } & \text { this } & \text { have.COND.1PL must.PP } & \text { say.INF=him.DAT }\end{array}$

'Why should we have said this to him?'

From a syntactic viewpoint, Rizzi's (2001a) analysis of perché in Italian why-questions is able to capture both exceptional behaviours in a straightforward way. Contrary to other whoperators, perché 'why' does not move from a position within the IP, but it is externally merged in a dedicated position in the left periphery, namely, the Spec of Int(errogative)P, above the landing site of the other bare wh-operators, that is, Spec/Foc: ${ }^{5}$

\section{(7) FORCE (TOP*) INT (TOP*) FOC $\quad$ (TOP*) FIN IP}

This positional difference, immediately supported by the ordering in (6), is also instrumental in capturing the observed difference with respect to inversion. Foc, attracting other wh-elements, is not inherently endowed with a $+Q$ feature. Therefore, it must acquire such a feature through

\footnotetext{
${ }^{5}$ Along the same lines, but with a slightly different implementation of this idea, Shlonsky \& Soare (2011) assume that why moves locally to Spec/Int from another left-periphery position. In embedded indirect yes-no questions, Int $^{\circ}$ hosts the interrogative complementizer corresponding to English if (see Rizzi 2001a).
} 
head movement of the inflected verb (assumed in the system of Rizzi 1996, 1997, 2001a to be a potential carrier of $+Q$ ), whence the obligatory inversion. On the contrary, Int is a position specialized for questions, hence it is inherently endowed with $+Q$. Therefore it does not trigger head movement of the inflected verb. Along these lines, the special position of why explains its compatibility with a preverbal subject (no inversion required), and its compatibility with a lower narrow focus (because the lower Foc head remains available to trigger focus movement or to license a focus in situ).

The derivation of a why-question (8) as opposed with a wh-question introduced by dove 'where' (9) is illustrated in (10) and (11), respectively:

Perché Gianni è partito?
why Gianni is left

'Why did Gianni leave?'

(9) Dov' è andato Gianni?

where is gone Gianni

'Where did Gianni go?'

(10) $\left[\mathrm{FP}\right.$ Force $\left[\right.$ IntP perché $\left[\right.$ Int $_{\mathrm{Q}}[\mathrm{FocP} \quad[\mathrm{IP}$ Gianni è partito $\left.\left.\left.]]\right]\right]\right]$ ?

(11) [FP Force $\left[\right.$ IntP Int [ FocP dove $e_{\mathrm{Q}}\left[\mathrm{I}^{\circ} \mathrm{è}\right] \mathrm{Q}[\mathrm{IP}$ pro $<\mathrm{è}>$ andato Gianni $<$ dove $\left.\left.\left.>]\right]\right]\right]$ ?

On the basis of Rizzi's analysis, we can thus expect two types of subject inversion in Italian wh-questions: (i) in why-questions, where subject inversion is not enforced by a syntactic constraint, we expect the possibility of the regular kind of subject inversion sensitive to information structure which is also found in Italian declaratives; as will be shown below (cf. $\S 3$ ), in this type of wh-questions the position of the subject depends on the information structure of the sentence, so that inversion is associated with a focal status of the subject, as is generally the case for the so called 'free' subject inversion in Italian (see Belletti 2004); (ii) with the other bare wh-operators, subject inversion is triggered by a specific syntactic requirement of the construction, and information structure plays no role.

In the following section we present the result of the syntactic experiment described and discussed in Bianchi, Bocci \& Cruschina (2017) and that was specifically designed to test this distinction. 


\section{Subject inversion in why-questions}

In order to investigate the focal nature of postverbal subjects in why-questions, Bianchi, Bocci \& Cruschina (2017) carried out a web-based two-alternative forced choice experiment with 64 native speakers of Italian. The experimental material consisted of 72 written fictional scenes introduced by a brief description and the task was to choose the sentence that sounded more natural in the provided context between two alternatives that only differed with respect to the position of the subject: preverbal vs postverbal. Three conditions were tested: (i) why-questions in neutral contexts (12), (ii) why-questions in contexts that favoured a focus interpretation of the subject (13) and (iii), as a control condition, wh-questions introduced by other bare whadjuncts - dove 'where' and come 'how'- presented in a neutral context (14), where the context was in fact the same as in the first condition with why-questions:

(12) Neutral context (broad focus):

[A causa di un problema tecnico hanno dovuto spostare la prova generale e le aule per le prove individuali sono state riassegnate, per cui Giulia chiede al direttore: 'Because of a technical problem the dress rehearsal was postponed and the rooms for the individual rehearsals have been reallocated, so Giulia asks the director:']
a. Perché Stefano balla?
why Stefano dances
b. Perché balla Stefano?
why dances Stefano

'Why is Stefano dancing?'

(13) Contexts favouring subject focalization (narrow focus):

[Giulia non sa che hanno cambiato il primo ballerino per il pas à deux e chiede stupita: 'Giulia doesn't know that the lead dancer for the pas à deux has been replaced and, surprised, asks:']
a. Perché Stefano balla?
why Stefano dances
b. Perché balla Stefano?
why dances Stefano
'Why is Stefano dancing?' 
(14) Neutral context (broad focus):

[ A causa di un problema tecnico hanno dovuto spostare la prova generale e le aule per le prove individuali sono state riassegnate, per cui Giulia chiede al direttore: 'Because of a technical problem the dress rehearsal was postponed and the rooms for the individual rehearsals have been reallocated, so Giulia asks the director:']
a. Dove Stefano balla?
where Stefano dances
b. Dove balla Stefano?
where dances Stefano
'Where is Stefano dancing?'

The results of this experiment are illustrated in Figure 1. As expected, postverbal subjects were almost always preferred in the control condition with dove 'where' and come 'how'. This suggests that subject inversion is obligatory in this condition, which was tested in neutral contexts. We can thus conclude that subject inversion in this condition is due to structural requirements and has nothing to do with information structure (see also Bianchi, Bocci \& Cruschina 2018 and Leonetti 2018).

Let us now turn to why-questions. In neutral contexts, that is, in the very same context as the control condition, we only observe a preference rate of $37 \%$ for subject inversion: preverbal subjects are indeed preferred in $63 \%$ of the cases. However, when the context is set so as to induce narrow focus on the subject, postverbal subjects are preferred in $66 \%$ of the cases, suggesting that the distribution of the subject in why-questions is indeed sensitive to information structure. Following Belletti (2004), we believe that the inverted focal subject (cf. e.g. in (13b)) targets a dedicated focus project in the periphery of the $\mathrm{vP}$. 


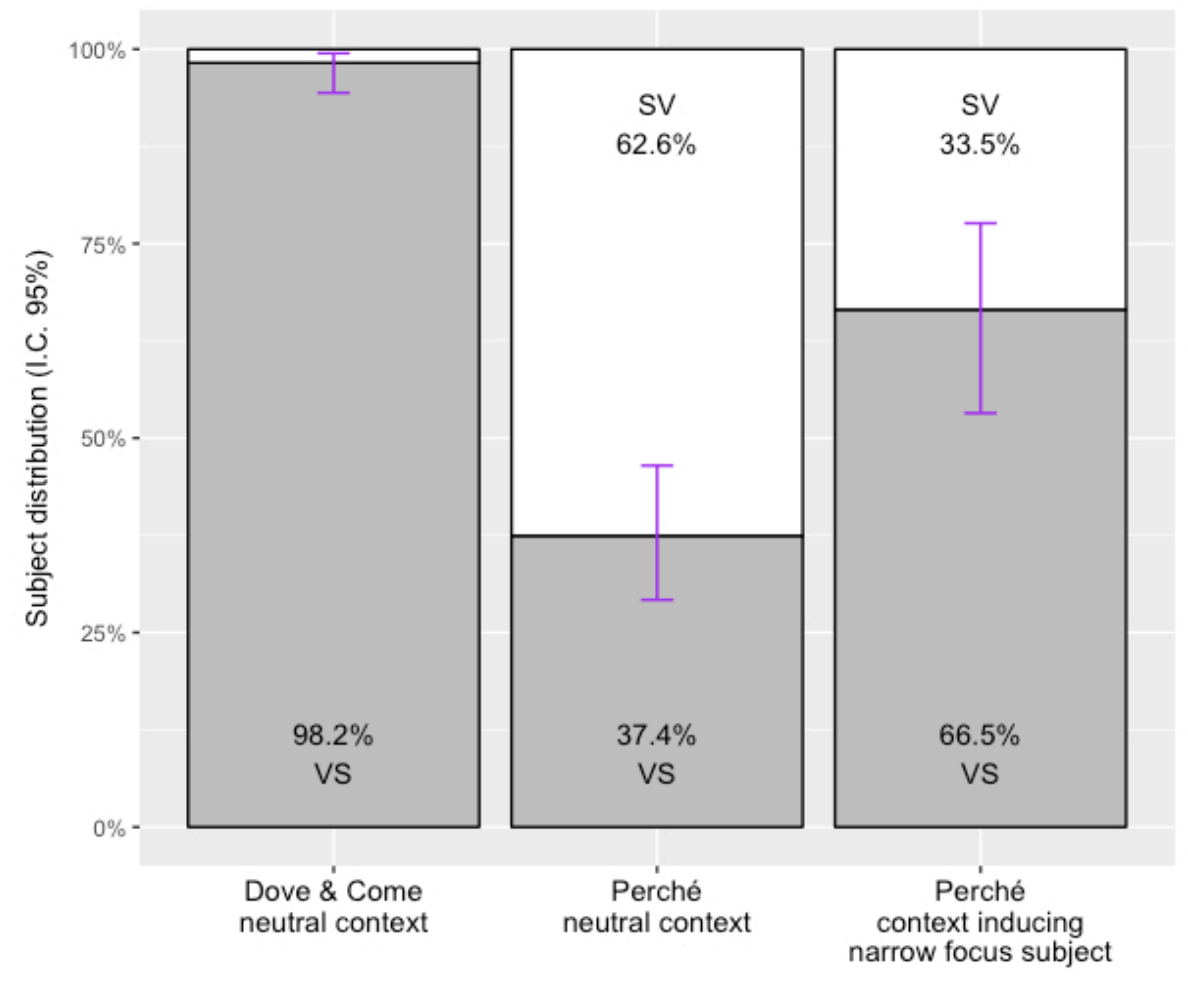

Figure 1: Preference for postverbal subjects (VS) over preverbal subjects (SV) across conditions (Bianchi, Bocci \& Cruschina 2017)

Summing up, Bianchi, Bocci \& Cruschina's (2017) experimental findings confirm that subject inversion is not obligatory in why-questions, as opposed to other types of wh-questions introduced by a bare wh-element. In addition, they show that, even if not mandatory, subject inversion is still possible in why-questions and that in that case, the position of the subject is to be related to its focal status. Let us now move to the prosodic properties of wh-questions.

\section{Prosodic properties of wh-questions in Italian}

To understand the special properties of why-questions at the prosodic level, we have to start from the general prosodic behaviour of wh-questions. In Italian, main prominence is by default assigned rightmost (Nespor \& Vogel 1986, Avesani 1990). This can be observed in neutral, broad focus, declarative sentences, where the rightmost constituent bears main phrasal stress and the nuclear pitch accent (NPA). In direct wh-questions, however, the NPA distribution exhibits a deviant pattern. In the presence of bare wh-elements, the NPA neither falls on the rightmost constituent, in contrast to the default pattern observed in declaratives, nor does it 
associate with the wh-element, as we may expect if the wh-element qualifies as focal. In bare wh-questions, the NPA is systematically assigned to a lexical verb. This is true of bare whquestions with short distance wh-movement where the wh-element is an argument of the matrix verb (15a), which bears the NPA. In cases of long distance wh-movement where the wh-word is extracted from an embedded clause (15b), the embedded verb bears the NPA (see Calabrese 1982, Ladd 1996, Marotta 2001, 2002, Bocci, Bianchi \& Cruschina 2020).

a. Short-distance movement

Chi pensa che ti dovrei presentare al direttore?

who thinks that you.DAT should.1SG introduce to-the directot

'Who thinks that I should introduce you to the director?'

b. Long-distance movement

$\begin{array}{llllll}\text { Chi pensi che dovrei } & \text { presentare al } & \text { direttore? } \\ \text { who think.2SG that } & \text { should.1SG introduce to-the } & \text { director }\end{array}$

'Who do you think that I should introduce to the director?'

Bocci, Bianchi \& Cruschina (2020) propose to link the NPA-assignment in Italian wh-questions to the successive cyclic nature of wh-movement. Wh-movement must pass through the edge of every $v \mathrm{P}$ and $\mathrm{CP}$ phase between the base-generation position and the final landing site in the left periphery of the sentence. In direct wh-questions, moreover, an interrogative wh-phrase bears a [wh/focal] feature, which can be viewed as a feature bundle. When the wh-phrase passes through the edge of a phase $\left(\mathrm{v}^{\circ}\right.$ or $\left.\mathrm{C}^{\circ}\right)$, it shares the $[\mathrm{wh} /$ focal] feature with the relevant phase head. ${ }^{6}$ As for the principles of the syntax-prosody interface that determine the mapping between syntactic and prosodic structure, Bocci, Bianchi \& Cruschina (2020) assume that the following rules are at the basis of the algorithm responsible for the NPA assignment:

(16) i. The NPA must be assigned to an element that is phonologically overt (and nonclitic; see Calabrese 1982, Nespor \& Vogel 1986). Thus, among the wh-copies in a

\footnotetext{
${ }^{6}$ This mirrors at the prosodic level what is expressed morpho(phono)logically in "wh-agreement" constructions in languages like Chamorro (Chung 1998) and Welsh (Willis 2000). More specifically, the NPA is assigned to the phase head (most typically, the lexical verb) adjacent to the intermediate position at the edge of vP through which the wh-phrase moves. Note also that, for Bocci, Bianchi \& Cruschina (2020), the precise agreement process is immaterial for the purposes of their analysis, that is, they do not commit themselves as to whether the whole feature bundle acts as a probe, or rather whether the [focus] feature is transmitted by the wh-phrase to the phase head via dynamic agreement in the sense of Rizzi (1996).
} 
wh-movement chain, only the highest copy is eligible for NPA assignment, the lower ones being subject to phonological deletion.

ii. When the syntactic structure contains one or more occurrences of the [focus] feature, the NPA must be assigned to a syntactic element that is marked with this feature (irrespective of whether the feature is interpretable or not on that element).

iii. The NPA is assigned to the rightmost element that satisfies (i) and (ii). If the sentence does not contain any occurrence of the [focus] feature, the NPA is assigned to the rightmost element by default (see Katz \& Selkirk 2011).

In other words, at the syntax-prosody interface, the NPA is assigned to the rightmost element in the sentence that is endowed with the [wh/focal] feature and that is not phonologically null.

A question featuring short-distance movement such as (15a) is thus analysed as illustrated in (17). The wh-phrase starts off from within the vP of the matrix clause and only shares its [wh/focal] feature with the phase heads in the matrix clause. Crucially, the $v^{0}$ and $\mathrm{C}^{0}$ heads of the embedded clause do not bear the [focus] feature, and hence they do not qualify for NPA assignment. Since traces are phonologically deleted, according to principle (16i) they are not possible targets for NPA assignment. As a consequence, the rightmost phonologically-realized element that is specified for the [wh/focal] feature is the matrix lexical verb pensa 'thinks', as indicated by the arrow ((17) is reproduced from Bocci, Bianchi \& Cruschina 2020: (28), without adapting the structure to the more cartographic representation adopted in the current paper; the same considerations hold for (18)):

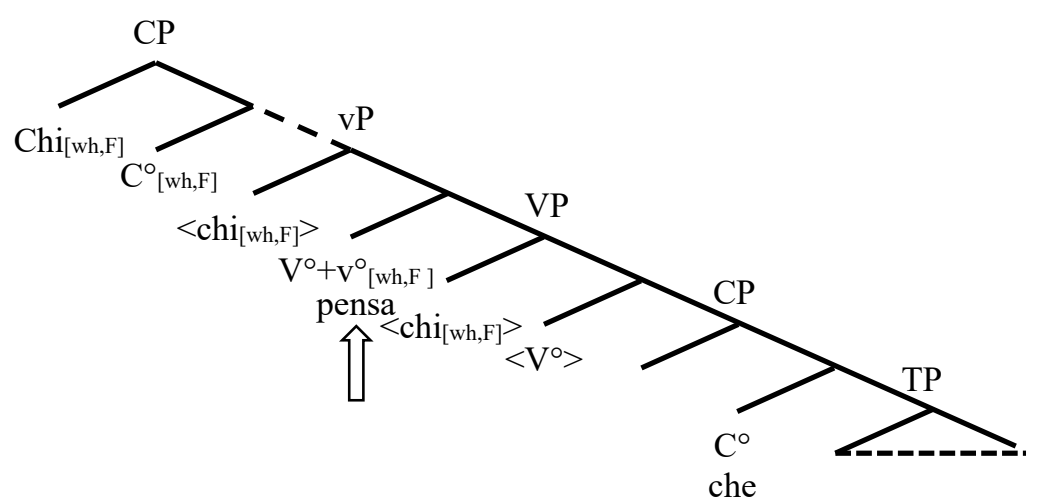


By contrast, in the case of long-distance movement like (15b), the wh-element is cyclically extracted from the $v \mathrm{P}$ of the embedded clause and, on its way to the $\mathrm{CP}$ of the matrix clause, it shares its [wh, focal] feature bundle with the head of each higher phase, as illustrated in (18):

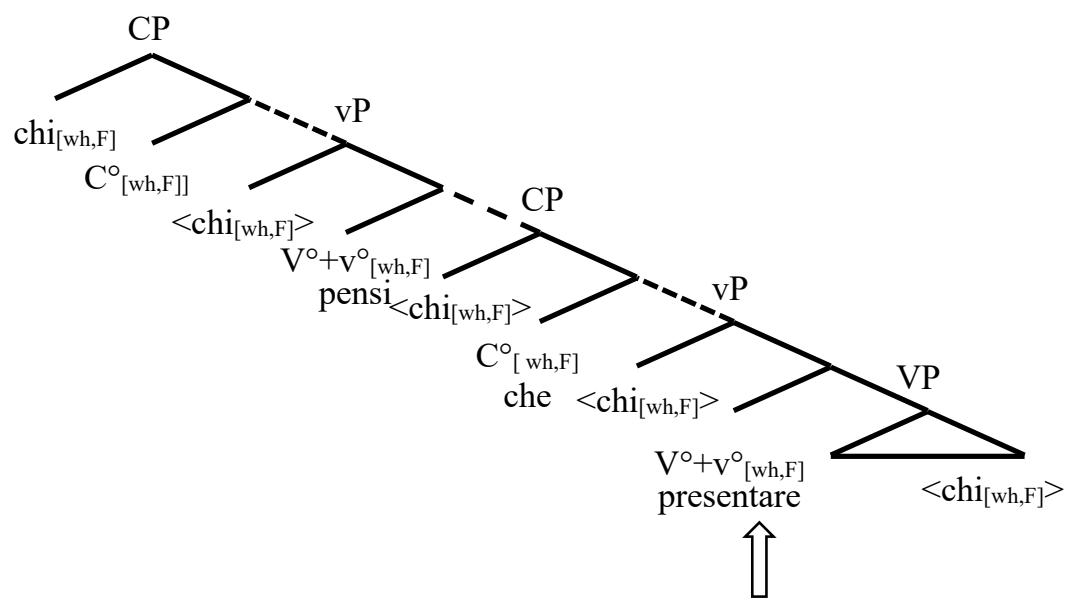

The rightmost phonologically realized position that is specified for the [focus] feature is the $v^{0}$ in the embedded clause. This head incorporates the matrix lexical verb, so the NPA is associated with the infinitive presentare 'introduce' which counts as the rightmost element endowed with the [wh/focal] feature and is not phonologically null.

This analysis predicts that when no wh-movement takes place, the [wh/focal] feature is not shared within the clause and there is thus only one element that is visible to the phonological component, that is, the interrogative wh-phrase itself, which bears a [wh/focal] feature. In this case, only principle (16ii) becomes relevant and the NPA must be assigned to a syntactic element that is marked with this feature. ${ }^{7}$ This prediction is indeed borne out by why-questions.

We saw that in bare wh-questions, the NPA is systematically assigned to the lexical verb, either to the matrix verb with short-distance movement or to the embedded verb in the cases of long-distance movement. ${ }^{8}$ Crucially, Marotta (2001) reports that direct questions with perché 'why' do no obey this generalization that holds for other bare wh-elements: in Italian whyquestions the NPA does not associate with the lexical verb, but rather with perché. At first sight, one might conjecture that this asymmetry between perché, on the one hand, and the other whelements, on the other, may result from the phonological weight that characterizes perché: being disyllabic and phonological heavy, it is able to bear main prominence, while phonological lighter elements fail to do so. However, as originally discussed by Marotta (2001) Italian has

\footnotetext{
${ }^{7}$ We return to the application of the algorithm for the NPA assignment in why-questions in Section 6.

${ }^{8}$ See Bocci, Bianchi \& Cruschina (2020) for a discussion of some degree of optionality, whereby in some instances of long-distance movement, the NPA may fall on the verb of the matrix clause.
} 
other disyllabic wh-elements (like quando 'when', dove 'where', come 'how', cosa 'what') and they, unlike perché, pattern with monosyllabic, light elements like chi 'who' and che 'what'.

Pushing forward this line of reasoning, Bocci (2013: § 6.7) advances another argument to reject the hypothesis that phonological weight may account for the peculiar patterns observed in bare-wh questions with why and the other wh-elements. He proposes the generalization that in aggressively non-D-linked wh-questions, the NPA cannot naturally associate with the whelement, but rather targets the lexical verb, as in case of bare wh-questions, whereas in D-linked wh-questions, the wh-phrase tends to attract main prominence, as in why-questions. For aggressively non-D-linked wh-questions and D-linked questions it is possible to construct near minimal pairs in which the wh-element features are equivalent in terms of phonological weight, that is, with respect to the numbers of syllables and presence of lexical stress. If different classes of wh-questions, but with similar phonological weight show opposed prosodic patterns, prominence distribution cannot be merely accounted for in terms of phonological weight.

In this paper, we propose that the same algorithm responsible for the assignment of the NPA in other wh-questions operate in the case of why-questions, but in the lack of whmovement from the IP, a different outcome is expected. As shown in the next section, the results of our prosodic experiment confirm this expectation and the opposition between why-questions and other wh-questions.

\section{NPA distribution in $w h y$-questions: The prosodic experiment}

To explore the prosodic properties of Italian why-questions, we carried out a production experiment in which we tested 8 native speakers of (Tuscan) Italian (two men and eight women from the area around Siena). The experiment consisted in a reading task.

We tested a single independent factor, 'wh-type' with three levels: (i) why-questions, (ii) D-linked wh-questions, and (iii) aggressively non D-linked wh-questions. A set of the experimental target sentences exemplifying the three conditions is given in (19).

a. Perché hai lavato il divano? (why-question)
why have.2sg washed the couch

'Why did you wash the couch?'

b. Chi di voi ha lavato il divano? (D-linked wh-question)
who of you have.3SG washed the couch
'Who of you washed the couch?'


c. Chi diavolo ha lavato il divano? (aggressively non D-linked wh-question) who devil has washed the couch

'Who the hell washed the couch?'

The D-linked wh-question and the aggressively non D-linked wh-question in each item formed a near-minimal pair, with an analogous number of syllables. Diavolo 'devil' and cavolo 'cabbage' are swear words in Italian and clearly feature lexical stress. In terms of phonological weight, chi diavolo and chi cavolo are somehow equivalent to wh-phrases of the type chi di loro 'who of you'. We created 5 items consisting of triplets analogous to (19) and we obtained 15 experimental stimuli.

The target sentences were inserted at the end of short fictional dialogues between two interlocutors. In order to minimize the impact of the linguistic context, for each item we used the same dialogue to introduce the target sentences. The dialogues were meant to introduce a 'neutral' wh-question. In order to use the same dialogues for different types of wh-questions and to preserve the coherence of the discourse, we used personal pronouns in the D-linked condition. This guaranteed the appropriateness of the discoursed-linked interpretation even if the previous dialogues did not mention a direct antecedent.

The factor 'wh-type' was manipulated within participant and within item. In order to prevent possible carry-over effects, we arranged the stimuli in 3 blocks so that within each block, each item was presented only once, under a single experimental condition. The 15 experimental stimuli were interspersed with 15 filler trials with an analogous dialogical structure. The order of the trials was pseudorandomized. Participants were asked to read aloud the dialogues, taking the role of both characters alternately. The sequence of the 30 trials were presented three times in order to collect three repetitions of each stimulus. No feedback was provided to the participants. If they reported that they felt unsatisfied with their production and wanted to repeat the production, we allowed them to read the sentence again. In these cases, we rejected the first production and we kept the new one. The recording took place individually in a quiet room in Siena (Italy). ${ }^{9}$ The experiment lasted between 45 and 60 minutes.

We collected and analysed a total of 352 target sentences ( 8 speakers $* 5$ items $* 3$ conditions $* 2 / 3$ repetitions). ${ }^{10}$ The sentences were segmented into phonemes and intonationally transcribed with a ToBi-like transcription system, within the theoretical framework of the

\footnotetext{
${ }^{9}$ To record the materials, we used a head-mounted microphone (Shure Beta 53) and a solid state recorder (Zoom $\mathrm{H}-4$ ) set a t $48 \mathrm{KHz}$ and 16 bits. The recordings were subsequently resampled to $16 \mathrm{KHz}$.

${ }^{10}$ We had to discard 8 sentences from the collected corpus because they featured clear segmental disfluencies.
} 
Autosegmental-Metrical Theory of intonation (Beckman \& Pierrehumbert 1986, Ladd 1996). The phonetic analyses were carried out using Praat (Boersma \& Weenink 2018) and the annotations were extracted via scripts.

The perceptual analysis of the sentences revealed that the target questions were all realized within a single intonational phrase. Moreover, the metrical head of the intonational phrase, i.e. the element endowed with main sentential stress was also assigned the Nuclear Pitch Accent (NPA), i.e. the most prominent pitch accent in the prosodic constituent. Following Gili Fivela et al. (2015: 156), we labelled as NPA the rightmost pitch accent after which the pitch contour is completely compressed and no subsequent fully-fledged pitch is observable. ${ }^{11}$ In what follows we will mainly discuss distribution of the NPA, but since its location coincides with that of main phrase stress, the same considerations hold true for the latter notion.

The results of this experiment concerning the distribution of the NPA in the three types of wh-questions are reported in Figure 2.1 As we can see, in all conditions, the NPA is never assigned to the rightmost constituent of the clause, that is, to the prominence default position in broad focus declaratives. In why-questions with perché, the NPA is virtually always assigned to the wh-element. In case of D-linked questions, we observe a partially similar pattern: the NPA is assigned to the wh-element in $85 \%$ of cases, while in the rest of the cases the NPA has been transcribed on the lexical verb $(12.8 \%)$ or not identified $(2.4 \%)$. In contrast with the two previous conditions, aggressively non-D-linked wh-elements behave like regular bare whquestions: the NPA is virtually never assigned to the wh-element (2.6\%), even though the whphrase is phonologically heavy; it is instead assigned to the lexical verb in the overwhelming majority of cases $(94.83 \%)$.

\footnotetext{
${ }^{11}$ In our data, no compressed PAs were identified in the post-nuclear region. As a consequence, the NPA in our data corresponds also to the rightmost PA before the right-boundary (and the possible edge tones) of the prosodic constituent.
} 


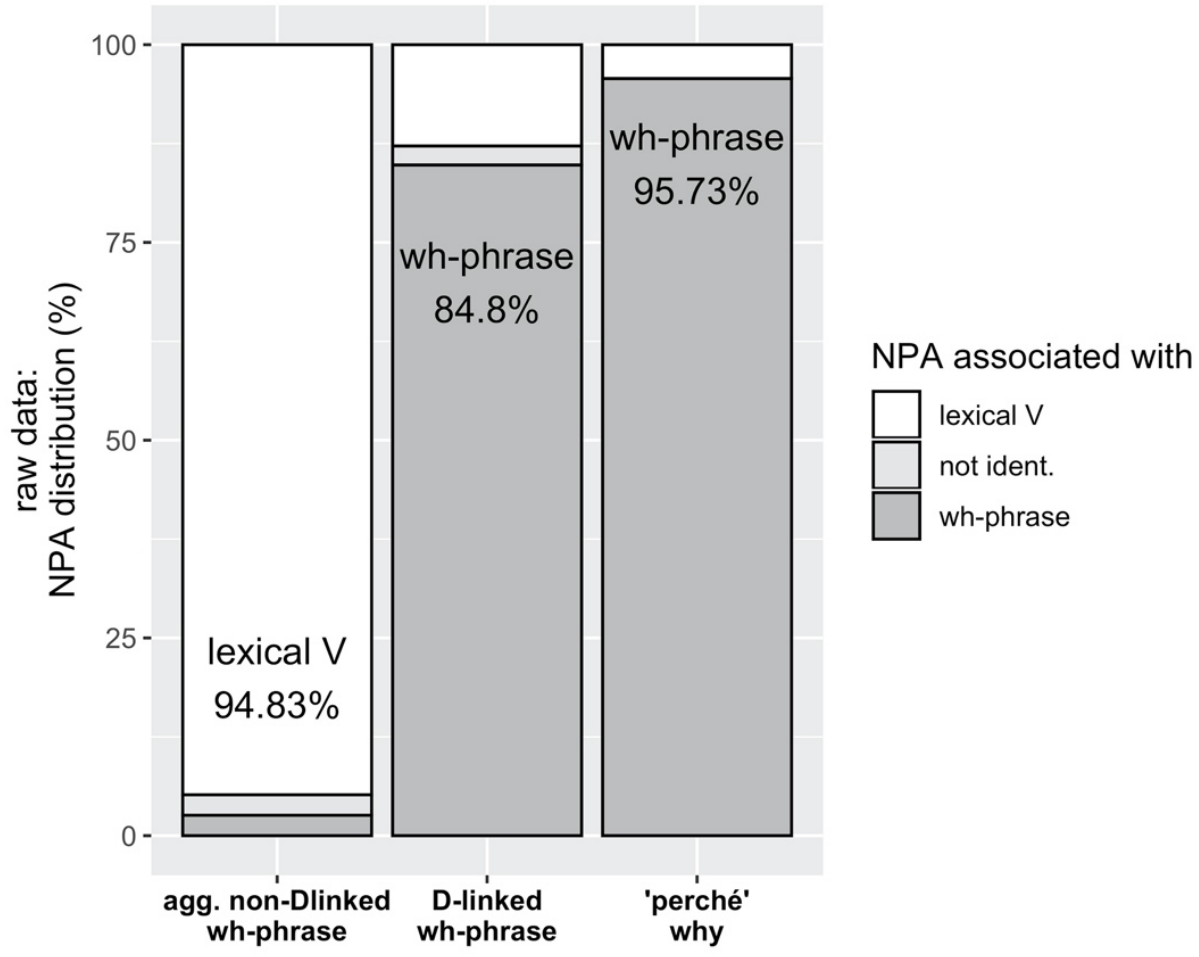

Figure 2.1: NPA distribution (\%) in direct wh-questions: aggressively non D-linked, Dlinked, and perché-questions.

To statistically test the NPA distribution across conditions, we excluded from the analysis the 6 sentences in which the NPA was not clearly identified. In this way, we could reduce the association site of the NPA to a binary variable: the NPA associated either with the wh-element or with the lexical verb. We built a multi-level mixed effects regression with the log odds of NPA on the wh-element as the dependent variable. We specified as independent factor the type of wh-questions, with a dummy coding, with aggressively non D-linked question as a reference category. The error structure included by-participant and by-item intercepts. The statistical test revealed that the NPA is significantly more likely to associate with the wh-element in D-linked questions (Est. 7.51, Std. Error 1.06, z value 7.07, p <.001) and in perché-questions (Est. 8.96, Std. Error 1.20 , $\mathrm{z}$ value $7.48, \mathrm{p}<.001$ ), rather than in aggressively non-D-linked questions. The coefficients extracted from the model are plotted in Figure 2.2 (along with the calculated confidence interval at $95 \%) .{ }^{12}$

\footnotetext{
${ }^{12}$ In Tuscan Italian, the presence of a high boundary tone in wh-questions is reported to be optional (Marotta 2001, Bocci, Bianchi \& Cruschina 2020). Our data confirm this observation: overall, a final rise L- $\mathrm{H} \%$ is observed in $50 \%$ of the data. In order to ascertain that the presence of the final rise is not related to the placement of the NPA,
} 


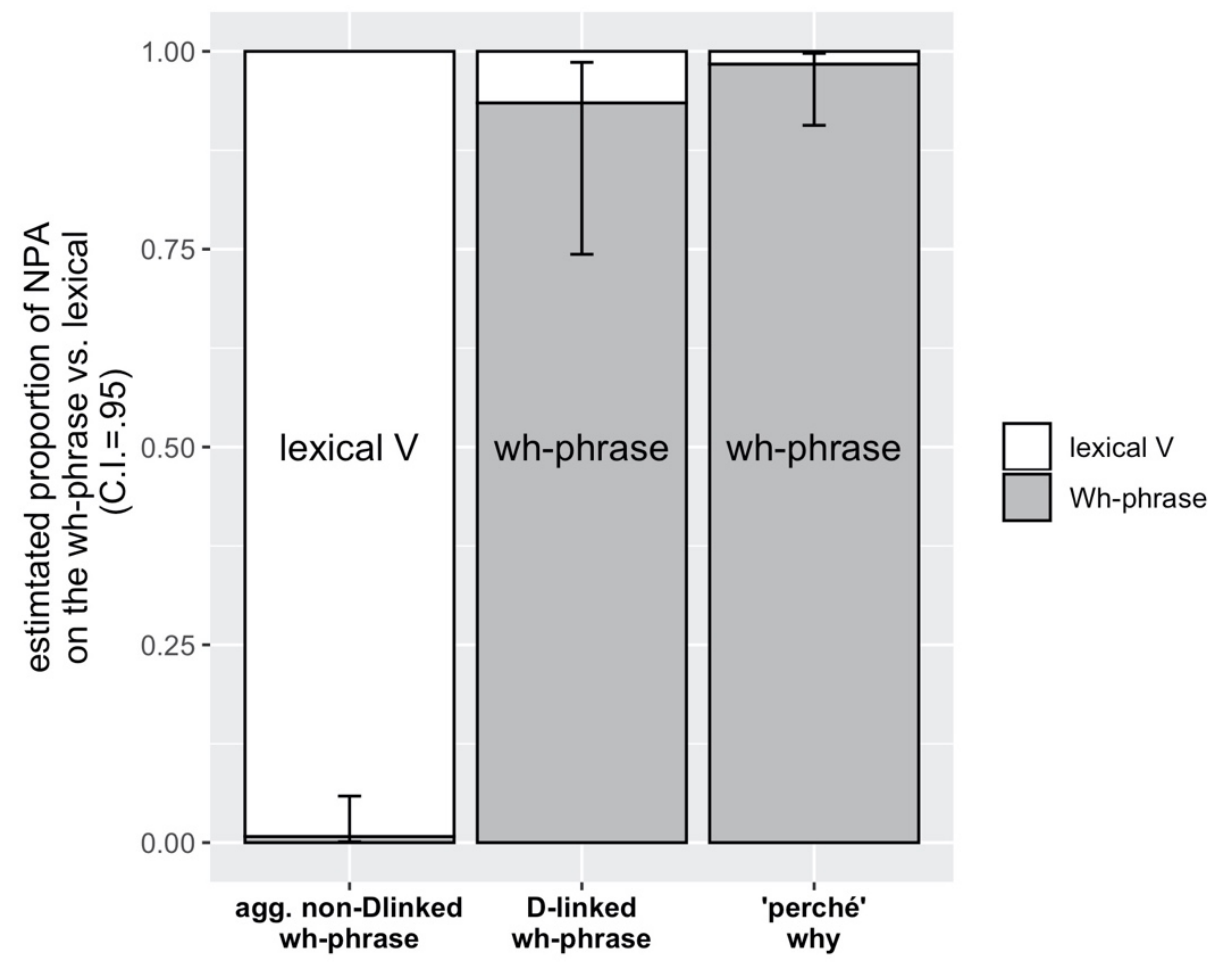

Figure 2.2: Estimated distribution of the NPA in direct wh-questions: aggressively non Dlinked, D-linked, and perché-questions.

Let us now consider some examples of pitch contours. As shown in Figure 3, in a why-question such as that in (19a), perché bears the NPA, the rightmost fully fledged pitch accent in this case (in this case, the only PA present in the sentence). More specifically, we observe that a $\mathrm{H}^{*}$ pitch accent is aligned with word-final stressed syllable of perché. No other pitch accent occurs in the rest of the clause. The only pitch movement we observe corresponds to the question final rise (resulting from the sequence of the phrase accent L- and the boundary tone $\mathrm{H} \%$ ) that culminates in the last (unstressed) syllable of the sentence-final word (i.e. divano).

we built a multi-level mixed effects regression with the NPA placement as a dependent variable and two independent factors: wh-type and presence of the final rise. The test showed that the type of wh-question is a significant predictor of NPA placement, while the presence of $\mathrm{L}-\mathrm{H} \%$ does not affect NPA distribution ( $\mathrm{z}$ value < 1). The interaction between final rise and wh-type also appeared to be non-significant. 


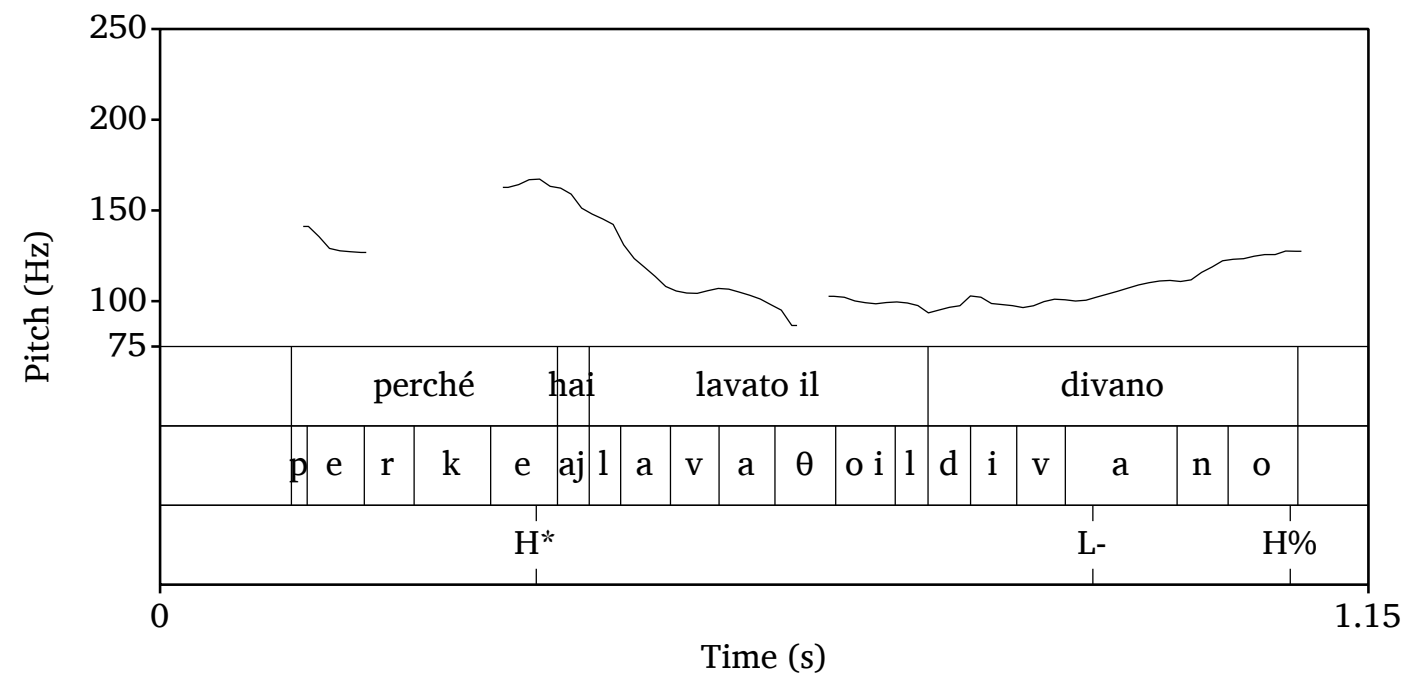

Figure 3: Pitch contour of an utterance produced after the why-question in (19a)

In the case of D-linked wh-phrases like (19b), the NPA is more likely to align with the whelement. This is shown in Figure 4, where $\mathrm{H}^{*}$, the only PA present in the sentence, is assigned to the complex wh-phrase chi divoi 'who of you'. After the NPA H* realized on voi, no other pitch accent occurs before final rise L-H\%.

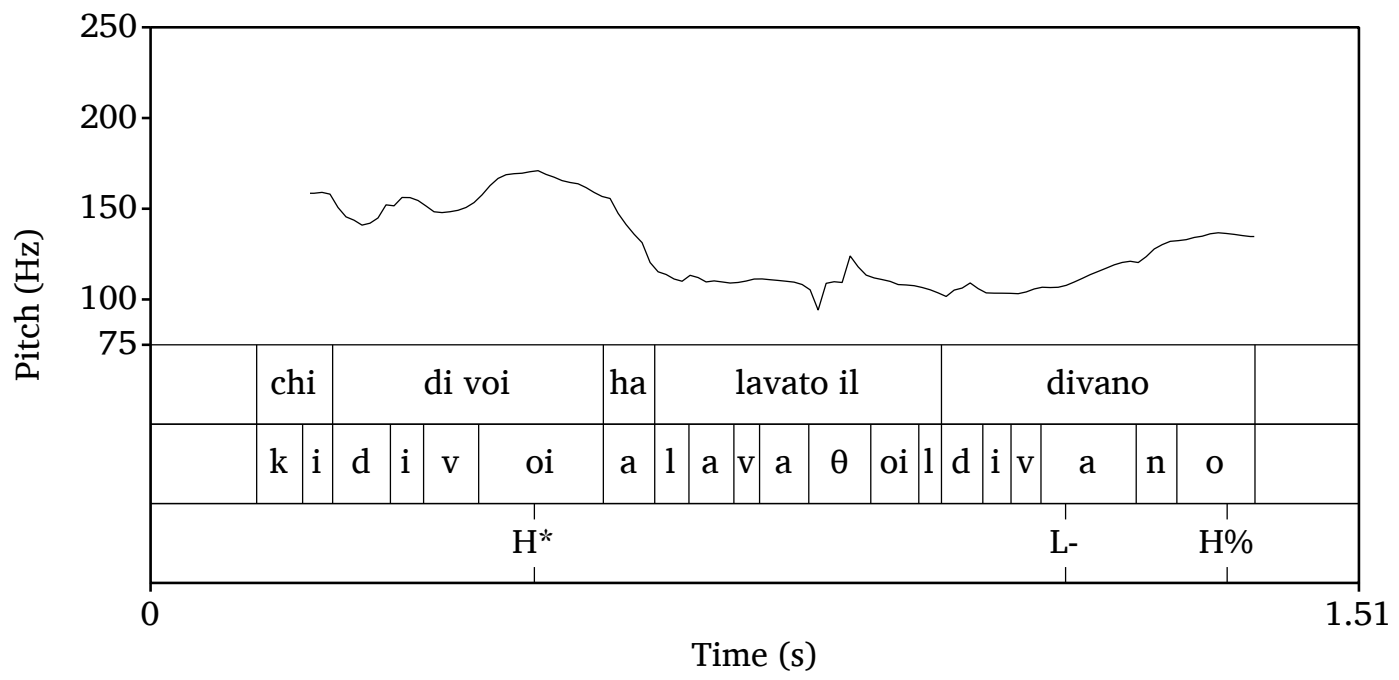

Figure 4: Pitch contour of an utterance produced after the D-linked wh-question (19b)

Finally, the pitch contour of an utterance produced after (19c) (see Figure 5) shows that in aggressively non-D-linked wh-questions behave like wh-questions introduced by bare wh- 
phrases, in that the NPA systematically falls on to the lexical verb: observe a NPA of the type $\mathrm{H}^{*}$ associated with lavato 'washed'.

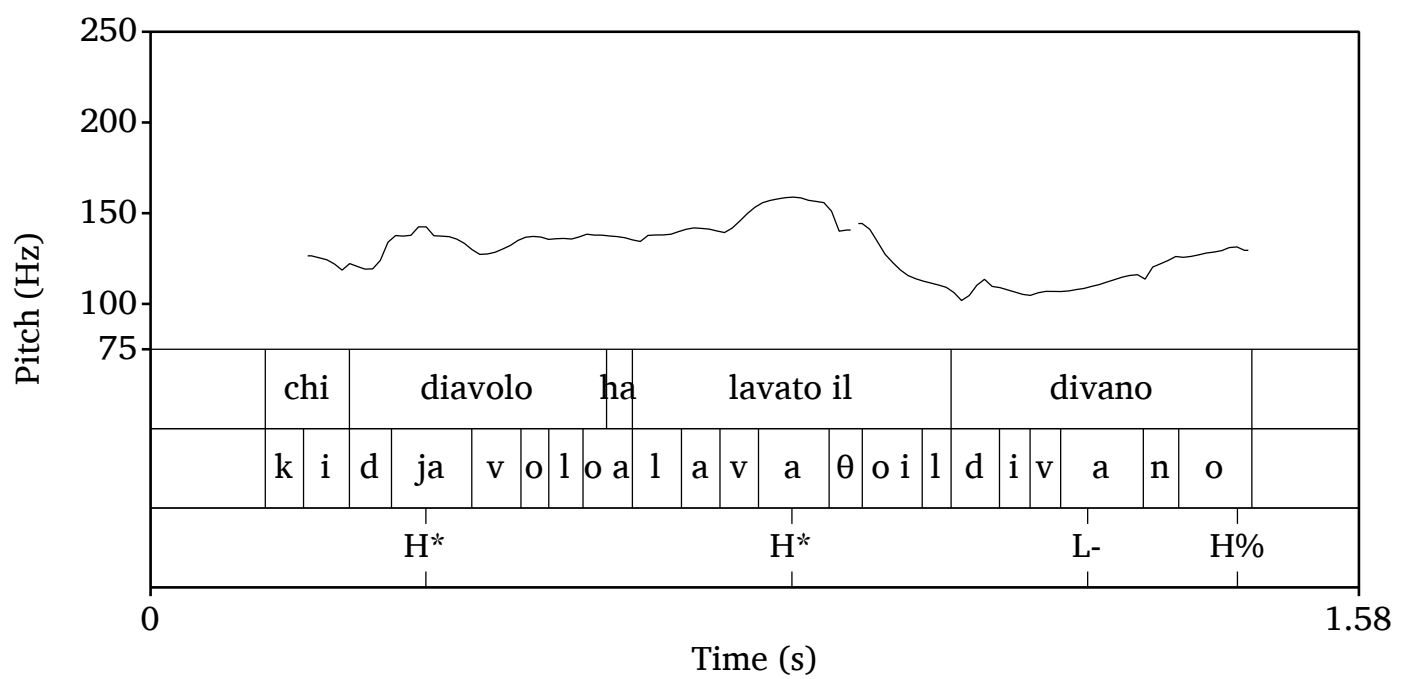

Figure 5: Pitch contour of an utterance produced after

the aggressively non-D-linked wh-question (16c)

In this paper we will not directly address and compare the prosodic behaviour of D-linked and aggressively non-D-linked wh-phrases, simply using them as terms of comparison with whyquestions, our main focus here. ${ }^{13}$ The results of our prosodic experiment suggest that in direct why-questions, why is typically associates with the NPA, much as D-linked wh-phrases ${ }^{14}$, and in contrast with aggressively non-D-linked wh-phrases. The sharp contrast between why and (phonologically complex) aggressively non-D-linked wh-phrases argues against an analysis of the property of why in terms of phonological weight, and calls for a syntactic explanation.

The explanation we offer to account for the NPA distribution in why-questions relies on a combination of the syntactic analysis by Rizzi (2001a) with the interface account by Bocci, Bianchi \& Cruschina (2020): unlike other wh-elements, perché 'why' does not undergo movement from a clause internal position and is externally merged in the left periphery (cf.

\footnotetext{
${ }^{13}$ A possible interpretation of the exceptional behaviour of D-linked wh-phrases that could be explored in future work is the following: when they pass through the edge of the vP phrase they do not share the focal feature with the phase head (cf. § 4). A further aspect that we leave to future work is that the similarity between D-linked whquestions and why-questions goes beyond the prosodic level and the assignment of the NPA: at the syntactic level, both D-linked wh-questions and why-questions do not strictly require subject inversion (see Rizzi 2001b).

${ }^{14}$ It is important to restrict this generalization to the context we tested, that is, to neutral contexts. Even if we did not include it as a further condition in our experiment, we expect that in perché-questions the NPA can associate with another constituent of the sentence for independent focalization purposes, provided the relevant contexts (cf. the syntactic experiment in $\S 3$ ).
} 
$\S 2)$. In why-questions, thus, the phase head little $v$ is not crossed over by perché and the lexical verb in little $\mathrm{v}^{\circ}$ then fails to attract NPA.

\section{The special prosodic behaviour of why}

In Section 4, we saw that in wh-questions with bare wh-elements other than why, the NPA is always assigned to the lexical verb and never to the wh-element itself. Why-questions, on the contrary, exhibit a different pattern: as shown in Section 5, the NPA predominantly falls on why itself. The special behaviour of why-questions can be taken to be the consequence of their different syntactic derivation. In other wh-questions the assignment of the NPA to the lexical verb is a reflex of the successive cyclic movement of the bare wh-element, which tracks the intermediate positions. Since the syntactic derivation of why is different from that of the other wh-elements, in that it does not undergo cyclic movement from a clause internal position but is directly merged in the left periphery, the asymmetry between why-questions and other whquestions is expected. We argue, however, that the algorithm responsible for the NPA assignment is the same for all wh-questions, including why-questions. The difference lies in the application conditions of the principles regulating the same algorithm. Let us consider again these principles, repeated here below as $\left(16^{\prime}\right)$ :

(16') i. The NPA must be assigned to an element that is phonologically overt (and nonclitic). Thus, among the $w h$-copies in a wh-movement chain, only the highest copy is eligible for NPA assignment, the lower ones being subject to phonological deletion.

ii. When the syntactic structure contains one or more occurrences of the [focus] feature, the NPA must be assigned to a syntactic element that is marked with this feature (irrespective of whether the feature is interpretable or not on that element).

iii. The NPA is assigned to the rightmost element that satisfies (i) and (ii). If the sentence does not contain any occurrence of the [focus] feature, the NPA is assigned to the rightmost element by default.

Condition (16i) is not relevant in the case of why-questions. Why is externally merged in the left periphery; since no wh-movement occurs, no wh-copies and chains are involved in the derivation of why-questions. For the same reasons, no feature sharing between perché and phrase heads can take place. The question to be addressed now is whether why-questions 
involve at least one occurrence of the focus feature, presumably born by why itself. This constitutes a precondition for the application of (16ii) and for preventing (16iii).

A positive answer to this question comes from a comparison with yes/no questions. In Italian neutral yes/no questions, when no narrow focus occurs, the NPA is assigned to the rightmost constituent of the sentence, namely, to the default prominence position of Italian (Gili Fivela et al. 2015). Since the derivation of yes/no questions does not crucially involve a focus feature (Bianchi \& Cruschina 2016), it is expected that prominence distribution in these structures patterns with what we observe in broad focus declaratives. Suppose now that no focus feature is present in why-questions: we would then expect the default prominence placement similar to that found in neutral yes/no questions and declarative clauses. As a matter of fact, however, our experimental findings and those reported in Marotta (2001) clearly show that neutral why-questions are characterized by a marked prosodic pattern in which the NPA is systematically assigned to perche' 'why', while the rest of the clause is prosodically subordinate to it. This marked prosodic pattern strongly suggests that a focus feature is assigned to perché, much as it is assigned to all other wh-elements. ${ }^{15}$ Algorithm (16) will thus assign NPA to perché itself, the rightmost overt element carrying [focus].

Exceptions to this general pattern of NPA assignment in why-questions are possible if an independent [focus] feature is present on another element of the syntactic structure. Recall that in Italian why-questions are compatible with a narrow focus, as shown in (20) (which repeats example (6) above):

$\begin{array}{lllll}\text { Perché } & \text { QUESTO } & \text { avremmo } & \text { dovuto } & \text { dirgli? } \\ \text { why } & \text { this } & \text { have.COND.1PL } & \text { must.PP } & \text { say.INF=him.DAT }\end{array}$

'Why should we have said this to him?'

This configuration is syntactically possible because perché and the fronted narrow focus do not compete for the same position, as would be the case with other wh-questions (Rizzi 1996, 1997). Prosodically, it is the narrow focus that associates with the NPA because it qualifies as the

\footnotetext{
${ }^{15}$ Alternatively, one could speculate that the marked prosodic distribution observed in neutral why-questions is not linked to the presence of focus, but rather to the fact that what follows perché is given/presupposed information and that as such must get destressed and deaccented. As a result, the NPA would surface on perché simply by virtue of the fact that perché would be the only element that is not given. There are two main arguments to reject this alternative hypothesis. First given information in Italian fails to be destressed prosodically (Bocci 2013 and references cited therein). Second, according to our intuitions, perché consistently associates with the NPA even when the proposition $p$ is clearly non-presuppositional, as in negative why-questions like (i):

(i) Perché non chiedi un congedo?

'Why don't you ask for a leave?'
} 
rightmost element that satisfies the interface principles in (16). In conclusion, why is the carrier of the [focus] feature and, as such, in neutral why-questions it acquires main prominence. But, if why cooccurs with another element bearing the [focus] feature, as in (20), the NPA is 'shifted' to that element, which is typically to its right (on the ban of the order Focus-Why, see Rizzi 2001a). ${ }^{16}$

\section{Conclusions}

The experimental studies reviewed in this paper clearly show that Italian why-questions behave differently from other types of wh-questions. At the syntactic level, why-questions do not require subject inversion and are compatible with a narrow focus, while at the level of the prosody, why typically associates with the NPA.

In our analysis, both properties can be accounted for if we assume that, unlike other whoperators, why does not involve wh-movement from IP and is directly merged within the left periphery. This syntactic difference is also at the basis of our account of the special prosodic behaviour of why-questions. In other wh-questions, the NPA associates with the lexical verb, a reflex of the syntactic derivation of the wh-element through the vP periphery before reaching its final landing site: the phase head $\mathrm{v}^{\circ}$ is the rightmost element carrying the [focus] feature, hence the lexical verb (ultimately associated with $\mathrm{v}^{\circ}$ via head movement) is assigned the NPA under (16). By contrast, the derivation of why involves no movement, so that the association of the NPA with a verbal element is correctly predicted not to hold. We have suggested that the assignment of the NPA to why itself also is the outcome of the syntax-prosody algorithm that assigns the NPA to the rightmost element in the structure that carries a [focus] feature. On the one hand, why stands out in that it is the only bare wh-element that is externally merged in the left periphery. On the other hand, like other wh-elements, and in opposition with the yes/no operator, why too is inherently assigned the [focus] feature. This determines the consequence that why carries the NPA in why questions like (19), under algorithm (16). As the results of our prosodic experiment show in section 5 , this prediction is correct. On the whole, the empirical findings discussed in this paper support the view that the syntactic structure guides

\footnotetext{
${ }^{16}$ This analysis raises the issue of how the [focus] feature on why satisfies the Focus Criterion if the focal head remains in a lower position. One possibility is that the Foc head may move to Int, and create the Spec-head configuration required for the satisfaction of the Focus criterion (in the case of (20) this would happen after the Foc head has satisfied the Focus Criterion for QUESTO). Alternatively, given the close association of Q and Foc in the wh-system, one could consider the possibility that the satisfaction of the Q Criterion by why in Spec-Int suffices to also satisfy the criterial requirements of its [focus] feature. We intend to explore these options in future work.
} 
computational processes at the interfaces with meaning and sound, as in much cartographic work (Cinque \& Rizzi 2010, Rizzi \& Bocci 2017). In particular, some aspects of the syntactic structures directly condition phonological operations such as the assignment of the NPA.

\section{Acknowledgements}

We would like to thank Valentina Bianchi and the audience of SLE 2017 for their constructive comments and suggestions, as well as the editor of this volume, Gabriela Soare, for her assistance and guidance through the publication process.

\section{References}

Avesani, Cinzia. 1990. A contribution to the synthesis of Italian intonation. Proceedings of the 1st International Conference on Spoken Language Processing, vol. I: 833-836.

Beckman, Mary E. \& Janet B. Pierrehumbert. 1986. Intonational structure in Japanese and English. Phonology Yearbook 3: 255-309.

Belletti, Adriana. 2004. Aspects of the low IP area. In Luigi Rizzi (ed.), The Structure of IP and CP. The Cartography of Syntactic Structures, Vol. 2, 16-51. New York: Oxford University Press.

Bianchi, Valentina \& Silvio Cruschina. 2016. The derivation and interpretation of polar questions with a fronted focus. Lingua 170: 47-68.

Bianchi, Valentina, Giuliano Bocci \& Silvio Cruschina. 2017. Two types of subject inversion in Italian wh-questions. Revue roumaine de linguistique 62 (3): 233-252.

Bianchi, Valentina, Giuliano Bocci \& Silvio Cruschina. 2018. The syntactic and prosodic effects of long-distance wh-movement in Italian. In Non-Canonical Postverbal Subjects, Delia Bentley \& Silvio Cruschina (eds), Special issue of Italian Journal of Linguistics 30(2): 59-78.

Bocci, Giuliano. 2013. The Syntax-Prosody Interface: A cartographic perspective with evidence from Italian. John Benjamins.

Bocci, Giuliano \& Lucia Pozzan. 2014. Questions (and experimental answers) about Italian subjects. Subject positions in main and indirect question in L1 and attrition. In Carla Contemori \& Lena Dal Pozzo (eds), Inquiries into Linguistic Theory and Language Acquisition. Papers offered to Adriana Belletti, 28-44. Siena: CISCL Press.

Bocci, Giuliano \& Silvio Cruschina. 2018. Postverbal subjects and nuclear pitch accent in Italian wh-questions. In From Sounds to Structures. Beyond the Veil of Maya, Rorberto Petrosino, Pietro Cerrone \& Harry van der Hulst (eds), 467-494. Berlin: De Gruyter. 
Bocci, Giuliano, Valentina Bianchi \& Silvio Cruschina. 2020. Focus in wh-questions: Evidence from Italian. Natural Language \& Linguistic Theory (E-pub ahead of print). DOI: https://doi.org/10.1007/s11049-020-09483-X

Boersma, Paul \& David Weenink. 2018. Praat: doing phonetics by computer [Computer program]. http://www.praat.org

Calabrese, Andrea. 1982. Alcune ipotesi sulla struttura informazionale della frase in Italia- no e sul suo rapporto con la struttura fonologica. Rivista di Grammatica Generativa 13: 489526.

Cardinaletti, Anna. 2007. Subjects and wh-questions. Some new generalizations. In Jose Camacho et al. (eds.), Romance linguistics 2006: Selected papers from the 36th Linguistic Symposium on Romance Languages (LSRL), 57-79. Amsterdam/Philadelphia: John Benjamins.

Chung, Sandra. 1998. The Design of Agreement: Evidence from Chamorro. Chicago: University of Chicago Press.

Cinque, Guglielmo \& Luigi Rizzi. 2010. The cartography of syntactic structures. In Heine Bernd and Narrog Heiko (eds.), The Oxford Handbook of Linguistic Analysis. Oxford: Oxford University Press, 65-78.

Gili Fivela, Barbara, Cinzia Avesani, Marco Barone, Giuliano Bocci, Claudia Crocco, Mariapaola D’Imperio, Rosa Giordano, Giovanna Marotta, Michelina Savino \& Patrizia Sorianello. 2015. Intonational phonology of the regional varieties of Italian. In Intonation in Romance, ed. Sonia Frota and Pilar Prieto, 140-197. Oxford: Oxford University Press.

Ladd, D. R. 1996. Intonational phonology. Cambridge University Press.

Laka, Itziar (1990). Negation in Syntax: On the Nature of Functional Categories and Projections. Doctoral dissertation. Cambridge, MA: MIT.

Leonetti, Manuel. 2018. Two types of postverbal subject. In Non-Canonical Postverbal Subjects, Delia Bentley \& Silvio Cruschina (eds), Special issue of Italian Journal of Linguistics 30(2): 11-36.

Marotta, Giovanna. 2001. I toni accentuali nelle interrogative aperte (wh-) dell'italiano di Lucca. In Atti del II congresso di studi dell'Associazione Italiana di Linguistica Applicata, Camilla Bettoni, Antonio Zampolli \& Daniela Zorzi (eds), 175-194. Perugia: Guerra Edizioni.

Marotta, Giovanna. 2002. L'Intonation des énoncés interrogatifs ouverts dans l'italien toscan. In Speech Prosody 2002, International Conference.

Nespor, Marina \& Irene Vogel. 1986. Prosodic Phonology. Berlin: Mouton de Gruyter. 
Poletto, Cecilia \& Raffaella Zanuttini. 2013. Emphasis as reduplication: Evidence from si che/no che sentences. Lingua 128: 124-141.

Rizzi, Luigi \& Giuliano Bocci. 2017. The left periphery of the clause: primarily illustrated for Italian. In The Wiley Blackwell Companion to Syntax, Second Edition, Martin Everaert \& Henk C. van Riemsdijk (eds). Oxford: Blackwell.

Rizzi, Luigi. 1996. Residual Verb-second and the Wh-criterion. In Adriana Belletti and Luigi Rizzi (eds), Parameters and Functional Heads. Essays in Comparative Syntax. Oxford/New York: Oxford University Press, 63-90.

Rizzi, Luigi. 1997. The fine structure of the left periphery. In Liliane Haegeman (ed.), Elements of Grammar, 281-337. Dordrecht: Kluwer.

Rizzi, Luigi. 2001a. On the position Int(errogative) in the left periphery of the clause. In Guglielmo Cinque \& Giampaolo Salvi (eds), Current studies in Italian syntax. Essays offered to Lorenzo Renzi, 287-296. Amsterdam: Elsevier.

Rizzi, Luigi. 2001b. Reconstruction, weak island sensitivity, and agreement. In Semantic Interfaces, Carlo Cecchetto, Gennaro Chierchia \& Maria Teresa Guasti (eds), 145-76. Stanford, CA: CSLI.

Shlonsky, Ur \& Gabriela Soare. 2011. Where's ‘why’?. Linguistic Inquiry 42(4): 651-669.

Stepanov, Arthur \& Wei-Tien Dylan Tsai. 2008. Cartography and licensing of wh-adjuncts: a cross-linguistic perspective. Natural Language \& Linguistic Theory 26(3): 589-638.

Willis, David. 2000. On the distribution of resumptive pronouns and wh-trace in Welsh. Journal of Linguistics 36: 531-573. 